\title{
A study on adverse drug reactions in hospitalized pediatric patients in a Tertiary Care Hospital
}

Arya Radhakrishnan Sindhu' ${ }^{1}$, Merin Sebastian ${ }^{1}$, Parvathy R. Panicker ${ }^{1}$, Sudha Muthusamy ${ }^{2 *}$, Venkateswaramurthy Nallasamy ${ }^{1}$, Sambathkumar Ramanathan ${ }^{3}$, Sattanathankaliya Perumal ${ }^{1}$

${ }^{1}$ Department of Pharmacy Practice, J.K.K.Nattraja College of Pharmacy, Kumarapalayam, India. ${ }^{2}$ Department of Pharmacology, J.K.K.Nattraja College of Pharmacy, Kumarapalayam, India.

${ }^{3}$ Department of Pharmaceutics, J.K.K.Nattraja College of Pharmacy, Kumarapalayam, India.

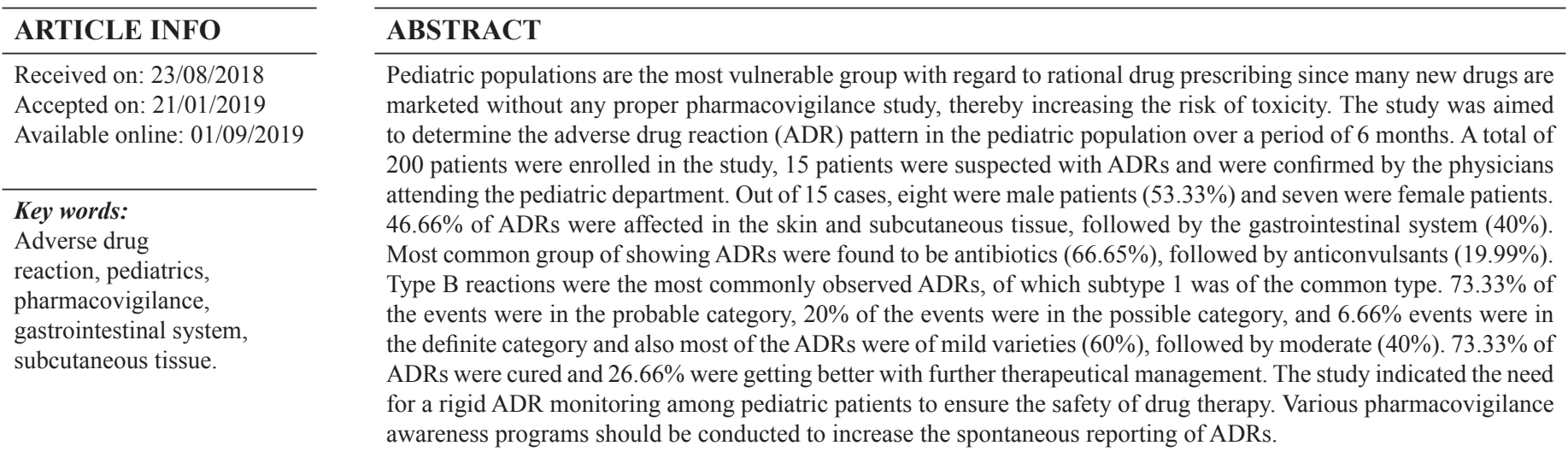

\section{INTRODUCTION}

Drug safety is an important issue in all medical disciplines. The problem of drug safety in pediatrics is compounded by the fact that medicines are often not tested in children, and therefore at the time of licensing, there is no indication for use in children. Due to developmental changes in physiology and drug handling prediction of the efficacy, dosing regimens and adverse drug reactions (ADRS) from adult data are inappropriate (Turner et al., 1993). It has long been an axiom in clinical pediatrics that "children are not just little adults," pediatrics population differ

\section{"Corresponding Author}

Sudha Muthusamy, Department of Pharmacology, J.K.K.Nattraja College of pharmacy, Kumarapalayam-638183, Tamilnadu, India.

E-mail: sudhacology@gmail.com from adults anatomically, physiologically, immunologically, psychologically, developmentally, and metabolically (Keams et al., 2003). Taken together with all these facts that the pattern of diseases in children is different from that in adults, this puts them at high risk of serious and unpredictable ADRs from the use of medicines.

The World Health Organization defines ADR as "a response to a drug which is noxious and unintended, and which occurs at doses normally used in man for the prophylaxis, diagnosis, or therapy of disease, or for the modification of physiological function." This definition excludes accidental or deliberate excessive dosage or maladministration (Raghavender et al., 2014).

Pharmacovigilance is the science related to compiling, monitoring, researching, qualifying, and evaluating the data obtained from healthcare professionals and patients about the 
adverse effects of drugs, biological and botanical products, as well as those used in traditional medicine (Agaard et al., 2010). The major aim of pharmacovigilance is to find out new adverse reactions to its documentation and accessing the observed ADRs. These reports include signals and expected reactions either during medical care, clinical research studies, intensive pharmacovigilance studies, and vaccination campaigns (Malcolm et al., 2015; Wasser fallen et al., 2001). In clinical research studies, suspected ADRs must be reported by the research centers and the sponsoring pharmaceutical company. The nature and the severity of signals are not mentioned in any of the scientific literature or in the information contained in product labeling, prescription documentation, or in the registration data, hence not possible to infer according to the pharmacological activity of the drug (Avery et al., 2011). Physicians, nurses, pharmacists, and pharmacy technicians are responsible for pharmacovigilance for children admitted to hospitals.

The perspectives of health-care professionals regarding what information parents require during episodes of suspected ADRs have not been described and the mechanisms for decision analysis and motivations which underpin the timing, content, and narrative of communication by clinicians have not been explored. In addition, there has been no attempt to identify if there are barriers to effective communication with families from the perspective of clinicians following a suspected ADR.

The safety of drugs used in patients of an adult group cannot be extrapolated to a pediatric age group. Both qualitatively and quantitatively, disease patterns in pediatrics populations differ from that of adults, which, in turn, may affect either the benefit or the risk of therapies (or both) with a resulting impact on the risk/benefit ratio (Kimland and Odlind, 2012; Tan et al., 2003). Significant variations are seen in the pharmacokinetics and pharmacodynamics of many commonly used drugs between pediatric and adults ward (Horen et al., 2002). The range of ADRs in children can differ from that of adults in terms of frequency, nature, and severity (Santos et al., 2008). The potential of ADR in children is high with a range of factors contributing to this vulnerability, including the physiological changes which take place from birth to late adolescence, lack of evidence-based information regarding the safety and/or efficacy of medicines for pediatric use, and high volume of off-label and unlicensed prescribing (Nahatae et al., 1999; Turner et al., 1999).

Pediatric populations are a more susceptible group with regard to rational drug prescribing since many new drugs are marketed without any proper pharmacovigilance study, thereby increasing the threat of drug toxicity (Mrutunjay et al., 2015). Also, ADR data in an adult cannot be relied upon to credit ADRs in children. In India, a study conducted in 2013 shows that incidence of ADR is between $5.9 \%$ and $22.3 \%$, while deaths due to ADRs account for $1.8 \%$. The US FDA and European medical agency emphasize the need to continually evaluate the beneficial and harmful effects of the drug in order to provide appropriate, safe, and effective drug therapies (Jha et al., 2013). Hence, the study was carried out to determine the occurrence of adverse drug reaction pattern in pediatric population that which helps the healthcare professionals to deal with the proper prescription of drugs.

\section{MATERIALS AND METHODS}

\section{Ethics approval}

The study was approved by the institutional ethics committee.

\section{Study design}

The study was a prospective, observational study, conducted in the inpatient department of a tertiary care hospital, Erode, Tamil Nadu, over a period a period of 6 months. A total of 200 cases were collected. The study included children of both sex and age group between 0 and 12 years and those hospitalized for more than 1 day.

All the cases were documented in the data entries and a questionnaire form was issued and filled by the parents or to the bystanders (from whom assent form was collected) regarding any history of allergic or unwanted reaction toward any drugs or foods. Patients were monitored for any ADR and the observed ADRs were documented. The observed ADRs were confirmed by the physician in charge or the staff nurse in charge. Causality and severity assessment was carried out using Naranjo's algorithm scale and Hartwig's scale, respectively. Further more, it was documented and the results were submitted.

\section{RESULTS}

A total of 200 patients were included in the study population, in which 15 patients were suspected with ADRs. The suspected ADRs were confirmed by the physicians attending the pediatric department.

A gender-wise distribution of ADR shows a male predominance, eight male patients $(53.33 \%)$ and seven female patients $(46.66 \%)$, it may be due to the fact that a number of admissions were seen in male children. During the study period, the maximum number of ADRs was seen among patients between 2 and 12 year's category, followed by infants, as more number of hospital admissions was under children category.

In our study, $46.66 \%$ of ADRs were affected in the skin and subcutaneous tissue, followed by the gastrointestinal system (40\%). Antibiotics were found to be associated with the occurrence of more number of ADRs (Fig. 1), in which amoxicillin showed more incidence of ADRs in the prescribed patients (Table 1). Type B was among the most commonly observed ADRs (Table 2). All the adverse drug reactions underwent causality and severity assessment using Naranjo's scale and modified Hartwing's scale, respectively (Fig. 2).

In case of outcome results, $11(73.33 \%)$ ADRs were cured and $4(26.66 \%)$ were getting better with further management. All the patients developed ADRs within a week of initiation of drug therapy. Thus, ADRs were quickly observed and were treated early (Table 3 ).

\section{DISCUSSION}

ADRs are an important clinical problem and contribute significantly to mortality and morbidity. ADRs in children can have a relatively more severe effect when compared to adults due to variation in pharmacokinetic and pharmacodynamic parameters within these two age groups. 


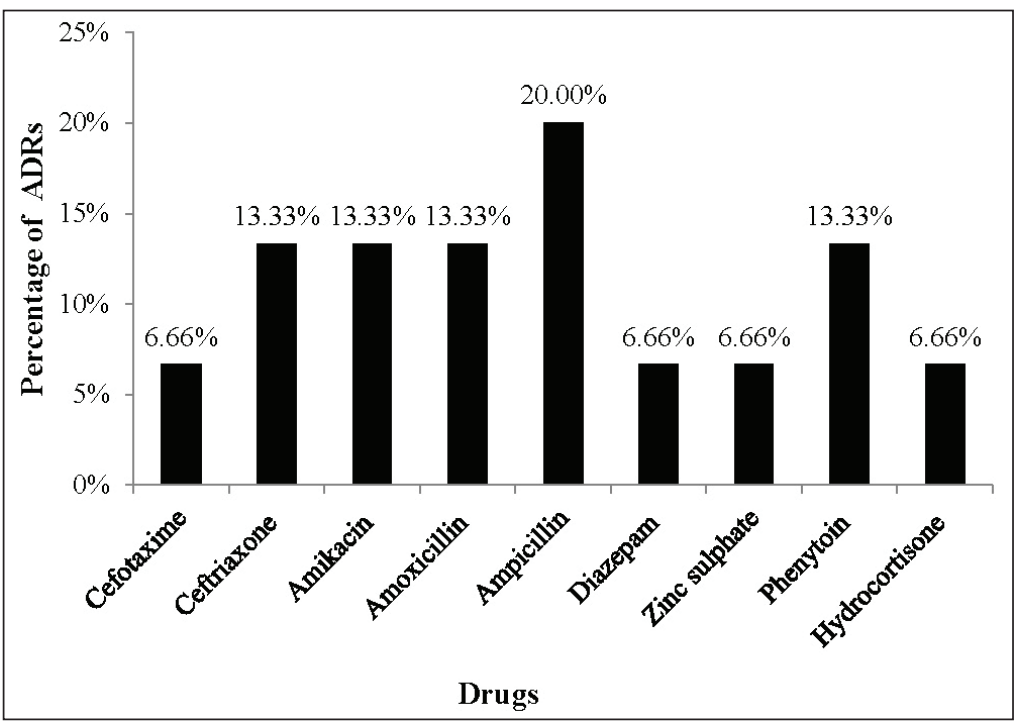

Figure 1. Drug-associated with the occurrence of ADRs.

Table 1. Incidence of ADRs among each drug in total population.

\begin{tabular}{clccc}
\hline SI. no. & Drug & $\begin{array}{c}\text { No of patients } \\
\text { prescribed }\end{array}$ & $\begin{array}{c}\text { No of ADRs } \\
(\boldsymbol{n}=\mathbf{1 5})\end{array}$ & $\begin{array}{c}\text { Percentage of } \\
\text { ADR occurred }\end{array}$ \\
\hline 1. & Cefotaxime & 72 & 2 & 2.77 \\
2. & Ceftriaxone & 32 & 2 & 6.25 \\
3. & Amikacin & 47 & 2 & 4.25 \\
4. & Amoxicillin & 5 & 2 & 40.00 \\
5. & Ampicillin & 34 & 2 & 5.88 \\
6. & Diazepam & 7 & 1 & 14.28 \\
7. & Zinc sulphate & 21 & 1 & 4.762 \\
8. & Phenytoin & 11 & 2 & 18.18 \\
9. & Hydrocortisone & 19 & 1 & 5.26 \\
\hline
\end{tabular}

Table 2. Categorization of the types of ADRs.

\begin{tabular}{clll}
\hline SI. no. & Suspected drug & Suspected ADR & Category \\
\hline 1. & Cefotaxime & Urticarial rashes & Type III \\
2. & Ceftriaxone & Lichen nitidus & Type IV \\
3. & Amikacin & Dry skin & Type A \\
4. & Amoxicillin & Urticaria & Type III \\
5. & Ampicillin & Itching on injection site & Type I \\
6. & Diazepam & Hypotension & Type A \\
7. & Zinc sulphate & Vomiting & Type I \\
8. & Phenytoin & Constipation & Type I \\
9. & Hydrocortisone & Itching all over the body & Type I \\
10. & Amikacin & dark bluish lip & Type A \\
11. & Cefotaxime & Mouth ulcer & Type IV \\
12. & Ceftriaxone & Diarrhea & Type I \\
\hline
\end{tabular}

The current prospective observational study focuses on the occurrence of ADR in the pediatric population and was carried out in a tertiary care hospital of Erode, Tamil Nadu. The result of the study showed a male predominance, as more number of hospital admissions was in the male group. This was dissimilar to that of a study conducted by Marius et al. (2001), where women had a higher number of ADRs. Women generally have a lower lean body mass, a reduced hepatic clearance, have differences in activity of CYP450 enzymes (40\% increase in CYP3A4, a varied decrease in CYP2D6, CYP2C19, and CYP1A2), and metabolize drugs at different rates compared with men. Increasingly the evidence was that gender difference in $T$ cell activation and proliferation account for this, as well as the increased prevalence of skin diseases in females. However, how these differences result in an increased risk of ADRs were not clear. A similar finding was depicted in a study where about $59.59 \%$ males and $40.41 \%$ females were found to be affected (Marius et al., 2011), which differ from the study by Mrutunjay et al. (2015), where 37.79\% ADRs and 62.20\% ADRs were reported for male and female patients, respectively.

The characteristics which facilitate the development of ADR in children were potentiated due to age-related divergents in physiological function, dissimilarity in disease pattern, and smaller size and body weight (Impicciatore et al., 2001), in our study more ADRs were found in 2-11 years old. This was similar to the study conducted by Mariapina et al. (2012).

If the patient body recognizes particular drug as an allergen, the immune system produces immunoglobin E specific to the drug and which triggers immediate hypersensitive reactions such as rashes, itching over the body. The most commonly seen skin reactions in our study were urticarial rashes, dry skin, dark bluish lips, and itching all over the body which was similar to the study conducted by Mrutunjay et al. (2015). A dissimilar study was found in Ruairi et al. (2010), where 53.21\% were affecting hematologic system followed by the gastrointestinal system $(32.13 \%)$.

In a study conducted by Jhaj et al. (1999), they have observed that beta-lactam group was involved with the highest incidence of cutaneous ADRs. In our study, among antibiotics, penicillin derivatives showed the maximum number of ADRs. Immediate prescription of antibiotics offers some benefits but balanced by increased cost, drug resistance, and adverse drug reactions. A practical approach of prescribing antibiotics is fulfilled 


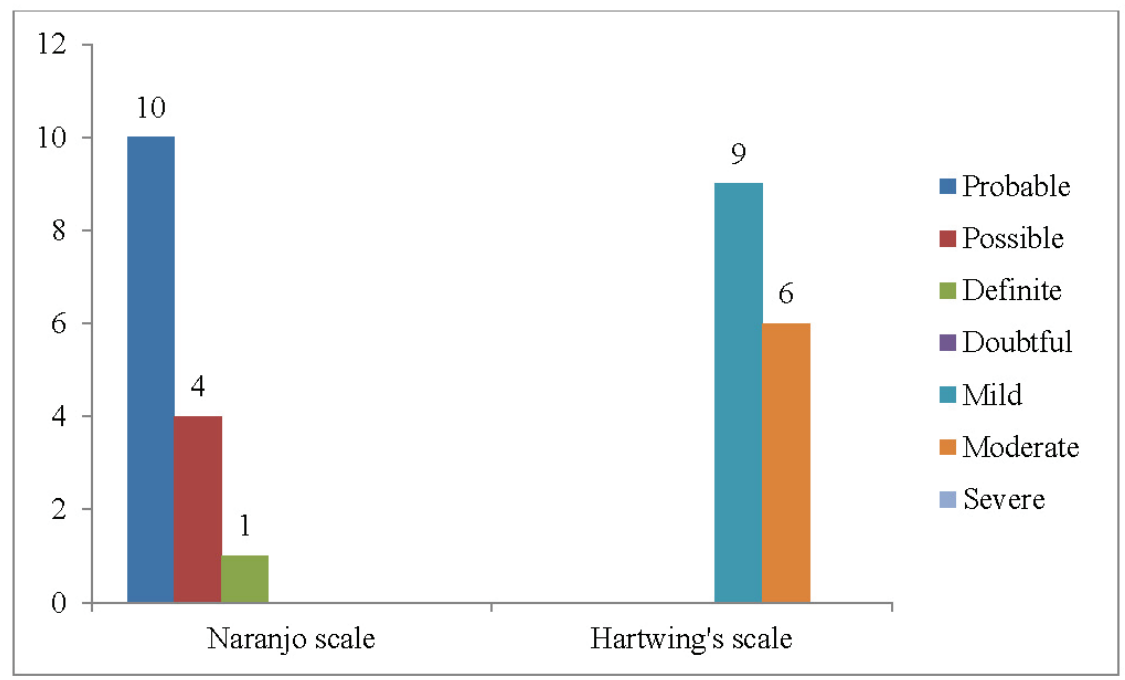

Figure 2. Causality and severity assessment of ADRs.

Table 3. Management of ADRs.

\begin{tabular}{|c|c|c|c|}
\hline Sl. no. & Suspected ADR & $\begin{array}{l}\text { No. of ADR } \\
\quad(n=15)\end{array}$ & Management \\
\hline 1. & Urticarial rashes & 2 & Stopped the suspected drug \\
\hline 2. & Lichen nitidus & 1 & Stopped the suspected drug \\
\hline 3. & Dry skin, dark bluish lip & 2 & Continued the treatment \\
\hline 4. & $\begin{array}{l}\text { Itching all over the body, } \\
\text { itching at the injection site }\end{array}$ & 2 & $\begin{array}{l}\text { Alternate medication is } \\
\text { given to prevent the reaction }\end{array}$ \\
\hline 5. & Constipation & 2 & Laxatives given \\
\hline 6. & Vomiting & 3 & Continued the treatment \\
\hline 7. & Hypotension & 1 & Dose reduction \\
\hline 8. & Diarrhea & 1 & $\begin{array}{l}\text { Continued the treatment } \\
\text { along with IV fluids }\end{array}$ \\
\hline 9. & Mouth ulcer & 1 & Continued the treatment \\
\hline
\end{tabular}

only if the child is not cured within 2-3 days of hospital admission or sooner if it appears that symptoms are worsening. This has been shown to feasible and acceptable, within a $76 \%$ reduction in the use of antibiotics (Jhaj et al., 1999). Malcolm et al. (2015) show one trial reported rates of diaper rashes and overall itching of about $50 \%$ among infants treated with amoxicillin-clavulanic acid.

In our study, out of five patients prescribed with amoxicillin, two were suspected with ADR (40\%) and urticaria was the commonly seen ADR. All forms of natural and semisynthetic penicillins can cause any allergic reactions, namely acute and subacute reactions mediated by $\operatorname{IgE}$ and $\mathrm{IgG}$ antibodies, respectively (Sanjib et al., 2010). Malcolm et al. (2015) conducted a metaanalysis and found that $27 \%$ of study subjects were found to be affected with candidiasis associated with amoxicillin and their study concluded that harms due to the drug were poorly reported in most trials, and their true incidence may have been higher than reported.

All drug-related adverse events were evaluated according to the Naranjo's probability scale and most of the events were in probable category. The study estimates that $5.6 \%$ of children treated with an antibiotic experienced cutaneous ADRs. However, they were rarely considered as serious. A similar study was found in Priyadharsini et al. (2011), which showed that most of the reactions had a probable causality score $(80 \%)$, followed by possible causality score $(20 \%)$. In their study, antibiotics were the major group associated with the ADRs (67\%) and cutaneous ADRs were the most common manifestations of such reactions $(37 \%)$.

By evaluating the ADRs using Hartwing's scale, most of the ADRs were of the mild category. Six ADRs were not serious, one ADR was serious, three ADRs resulted in prolonged hospitalization, and five ADRs required intervention to prevent that (Fig. 2). Mild or moderate adverse drug reactions do not necessarily mean that a drug must be discontinued, especially if no suitable alternative is available. Severe reactions include those that may be life-threatening (such as liver failure, abnormal heart rhythms, and certain types of allergic reactions), that results in persistent or significant disability or hospitalization, and that cause a birth defect (Mritunjay et al., 2015). The severe categories were of very few $(6.66 \%)$. The study was comparable to various previous results (Impicciatore et al., 2001).

Type B reactions were the most commonly observed ADRs. Among type B reaction, type 1 was most commonly seen and this was because of allergic reactions to beta lactamic drugs were more common. Drug reactions commonly manifested with dermatologic symptoms were caused by the metabolic and immunologic activity of the skin. The most common dermatologic manifestation of drug reaction was urticarial rashes. Typically, lichen nitidus rash appeared within 2-3 days after drug exposure, originated on the trunk, and eventually spread to the limbs. A similar study was depicted by Kishour et al. 2015, in which $83.6 \%$ of ADRs was type B, $14.42 \%$ was type A, and $1.92 \%$ was type $U$ and they state that most of the ADRs were inevitable and unavoidable. But Khan et al. (2015) found that proportion of augmented ADRs or type A ADRs were more and they state that these ADRs were predictable and therefore can be prevented by careful therapeutic selection of drugs and precedence of this should be taken by the healthcare providers in order to improve the cost-effectiveness and safety for these illnesses. In our study, two ADRs such as urticarial rashes and lichen nitidus required to stop the suspected medication. 
A number of serious reactions requiring hospitalization in our study was 1 around $6.66 \%$ of the total ADRs. They had undergone prompt management without any mortality report. In a recent single practice study, general practitioners estimated that the presenting symptom of $1.7 \%$ of their consultations over a 6-month period was a manifestation of an ADR. Furthermore, it was likely that $2 \%-6 \%$ of hospital admissions were for ADRs (Khan et al., 2006). Most of the studies undergone proper management without any mortality but in another study one death was reported. This was a case in which a 4-year-old male child diagnosed with status epilepticus was administered thiopentone sodium injection followed by which he had vomiting, skin rashes, and death (Priyadharsini et al., 2011). All the patients developed the ADRs within a week of initiation of drug therapy. Thus ADRs were quickly observed and thus treated early. Similar observations have been made before (Ramesh et al., 2003).

\section{CONCLUSION}

The methods for ADR detection, evaluation, and monitoring should be strengthened for the pediatric population. The role of pharmacovigilance in monitoring the safety of drugs in children should be evaluated in the detection of newer and rarer ADRs. The awareness of spontaneous reporting of ADRs among healthcare professionals and the general population should be given due considerations for preventing the morbidity and mortality among the pediatric population. Out of 200 pediatric patients, 15 ADRs occurred and were more among children category. Penicillin derivatives were the most commonly implicated drugs, which were tolerated by the patients followed by anticonvulsant agents. Most of the reactions were of moderate severity. ADR alert card was distributed to the caregivers of the patients affected by ADR. The study indicates the need for a rigid ADR monitoring among pediatric patients to ensure the safety of drug therapy. Various pharmacovigilance awareness programs should be conducted to increase the spontaneous reporting of ADRs.

\section{LIMITATION}

The study has the limitation of being a short-term study and a total of 200 cases were able to collect. Re-challenge and dechallenge were not able to perform due to ethical concerns and all the ADRs may not be well noticed in children as they are unable to express.

\section{ACKNOWLEDGMENTS}

We thank the Head of the department and departmental staff for the suggestions and support for completion of this work.

\section{REFERENCES}

Avery AJ, Anderson C, Bond CM, Lee AJ, Murphy E, Shakir $\mathrm{S}$, Watson MC. Evaluation of patient reporting of adverse drug reactions to the UK 'Yellow Card Scheme': literature review, descriptive and qualitative analyses, and questionnaire surveys. Health Technol Assess, 2011; 15(20):212-34.

Horen B, Montastruc JL, Lapeyre MM. Adverse drug reactions and off-label drug use in pediatrics outpatients. Br J Clin Pharmacol, 2002; 54(6):665-70.

Impicciatore $\mathrm{P}$, Choonara I, Clarkson A, Provasi D, Pandolfini C, Bonati M. Incidence of adverse drug reactions in paediatric in/out-patients: a systematic review and meta-analysis of prospective studies. Br J Clin Pharmacol, 2001; 2(1):51-77.
Jha K, Larizgoitia I, Audera-Lopez C, Prasopa-Plaizier N, Waters H, Bates DW. The global burden of unsafe medical care: analytic modelling of observational studies. BMJ, 2013; 22(10):809-15.

Jhaj R, Uppal R, Malhotra S, Bhargava VK. Cutaneous adverse reactions in in-patients in a tertiary care hospital. Indian J Dermatol Venerol Leprol, 1999; 65(1):14-17.

Kearns GL, Abdel-Rahman S, Leeder JS, Alander SW, Kauffiman RE. Developmental pharmacology-drug disposition, action, and therapy in infants and children. N Engl J Med, 2003; 349:1157-67.

Khan L, Le J, Nguyen AV, Hodding J. Adverse drug reactions among children over a 10-year period. Pediatr, 2006; 43(2):27-32.

Kimland E, Odlind V. Off-label drug use in pediatric patients. Clin Pharmacol Ther, 2012; 91(5):796-801.

Kishour KD, Aakash G. Pattern of adverse drug reactions in children attending the department of Pediatrics in a Tertiary Care Center: A prospective observational study. Clinical medicine insights. Pediatr, 2015 9(7):73-8.

Malcolm G, Kavita D, Akanksha S, Preeti G. Pattern of adverse drug reactions to antibiotics commonly prescribed in department of medicine and pediatrics in a tertiary care teaching hospital, Ghaziabad. JAPS, 2015; 5(4):78-82.

Mariapina G, Antonio C, Mauriziio B, Siani P, Rossi F, Capuano A. Active surveillance of adverse drug reactions in children in five Italian paediatric wards. Open J Pediatr, 2012; 2(2):111-17.

Marius R. Do Women Have More Adverse Drug Reactions? Am J Clin Dermatol, 2001; 2(6):349-51.

Mrutunjay D, Jena M, Mishra S, Panda M, Patro N. Monitoring of Adverse Drug Reactions in Pediatric Department of a Tertiary Care Teaching Hospital: A Hospital Based Observational Study. IJPRAS, 2015 4(4):69-76.

Priyadharsini R, Surendiran A, Adithan C, Sreenivasan S, Sahoo FK. A study of adverse drug reactions in pediatric patients. J Pharmacol Pharmacother, 2011; 2(4):277-80.

Raghavender R, Rohit S, Srawan D, Vijay R. A prospective review on pharmacovigilence. Int J Pharm Sci Invent, 2014; 2(2):653-658.

Ramesh M, Pandit J, Parthasarathi G. Adverse drug reactions in a south Indian hospital - their severity and cost involved. Pharmacoepidemiol Drug Saf, 2003; 12(8):687-92.

Ruairi M, Aagaard L, Weber CB, Hansen EH. Adverse drug reactions in the pediatric population in Denmark: a retrospective analysis of reports made to the Danish Medicines Agency from 1998 to 2007. Drug Saf, 2010; 33(4):327-39.

Sanjib B. The facts about penicillin allergy: a review. J Adv Pharm Technol Res, 2010; 1(1):11-17.

Santos DB, Clavenna A, Bonati M, Coelho HL. Off-label and unlicensed drug utilization in hospitalized children in Fortaleza, Brazil. Eur J Clin Pharmacol, 2008; 64(11):1111-18.

Tan E, Cranswick NE, Rayner CR, Chapman CB. Dosing information for pediatric patients: are they really "therapeutic orphans"? Med J Aust, 2003; 179(4):195-8.

Turner S, Nunn AJ, Fielding K, Choonara I. Adverse drug reactions to unlicensed and off-label drugs on pediatrics wards: a prospective study. Acta Paediatr, 1999; 88:965-68.

Wasserfallen J, Livio F, Buclin T, Tillet L, Yersin B, Biollaz J. Rate, type, and cost of adverse drug reactions in emergency department admissions. Eur J Intern Med, 2001; 12(5):442-47.

How to cite this article:

Sindhu AR, Sebastian M, Panicker PR, Muthusamy S, Nallasamy V5, Ramanathan R, Perumal S. A study on adverse drug reactions in hospitalized pediatric patients in a Tertiary Care Hospital. J Appl Pharm Sci, 2019; 9(09):072-076. 